\title{
Correction to: MicroRNA-335-5p is a potential suppressor of metastasis and invasion in gastric cancer
}

Alejandra Sandoval-Bórquez 1,2,3, Iva Polakovicova 1,3 , Nicolás Carrasco-Véliz 1,3,4, Lorena Lobos-González 5,6, Ismael Riquelme2, Gonzalo Carrasco-Avino 1,7, Carolina Bizama3, , Enrique Norero 9,10, Gareth I. Owen 1,3,11, Juan C. Roa ${ }^{1,2,3,8}$ and Alejandro H. Corvalán ${ }^{1,3,12^{*}}$

\section{Correction to: Clinical Epigenetics (2017) 9:114} https://doi.org/10.1186/s13148-017-0413-8

Following publication of the original article [1], the authors identified an error in Fig. 2. The correct figure is given below (Fig. 2). 

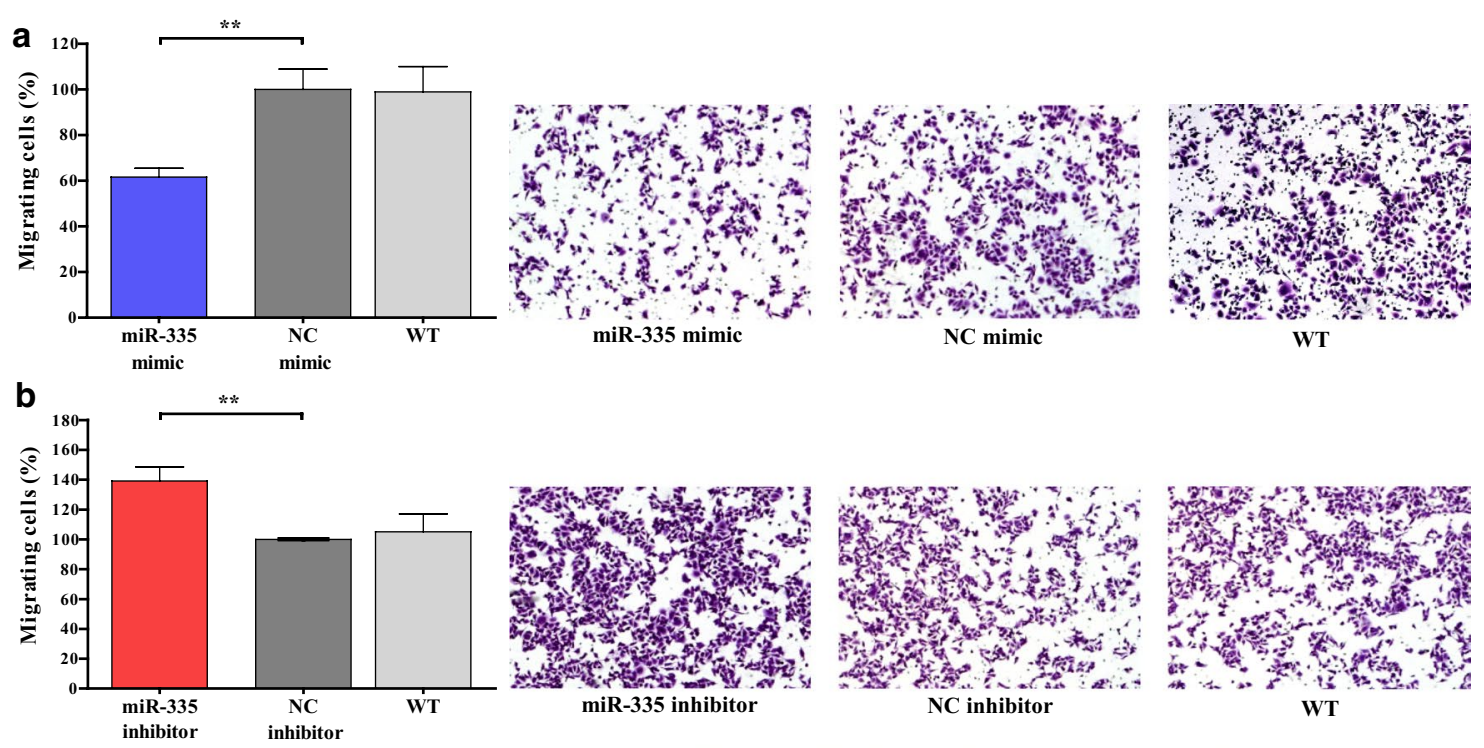

NC mimi

WT
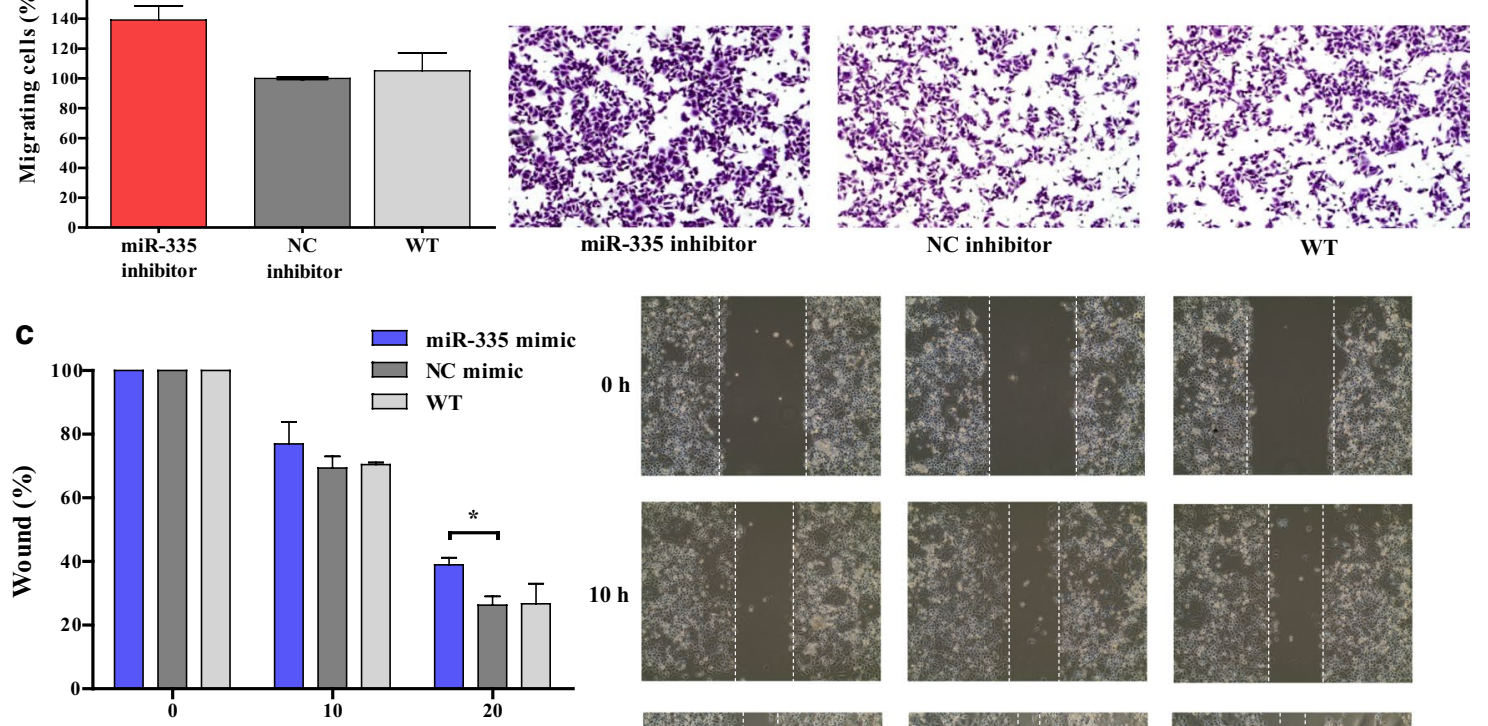

Time (hours)
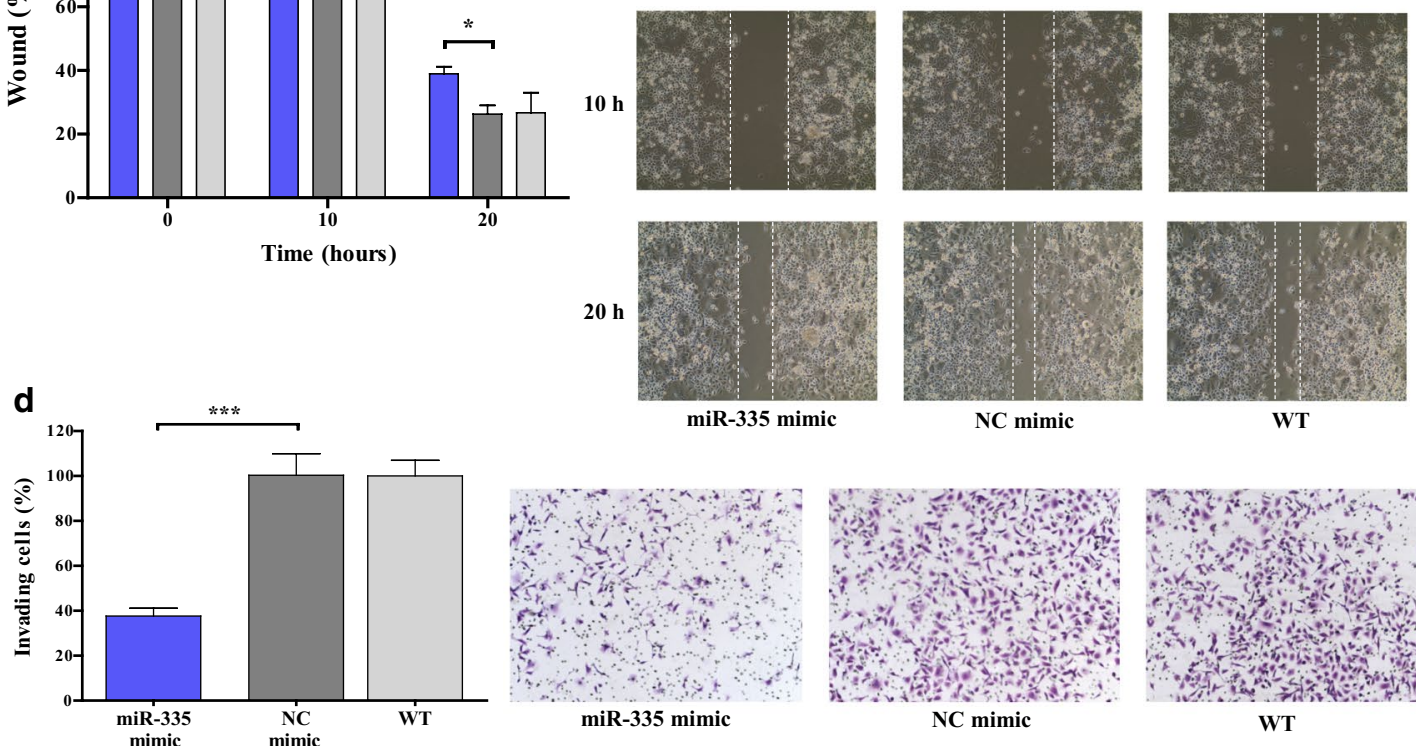

miR-335 mimic

NC mimic

WT
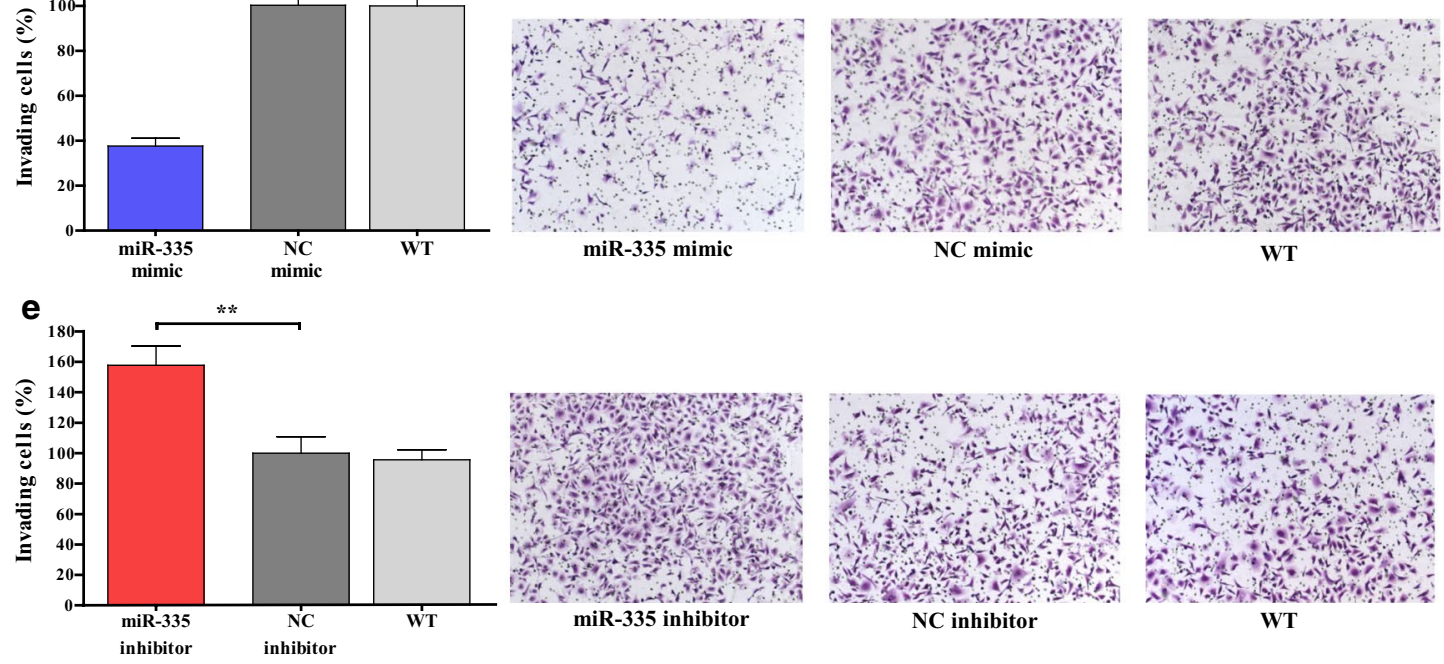
(See figure on previous page.)

Fig. 2 Overexpression of miR-335 inhibits cell migration and invasion. a, b Representative images of AGS cells transfected with NC/miR-335 mimic or with NC/miR-335 inhibitor in migration assay. c Representative images of AGS cells transfected with control NC/miR-335 mimic in wound healing assay. $\mathbf{d}$, e Representative images of AGS cells transfected with control NC/miR-335 mimic or with NC/miR-335 inhibitor in invasion assay. Results represent the means of three independent experiments; bars indicate SD. ${ }^{*} p<0.05,{ }^{* *} p<0.01,{ }^{* * *} p<0.001$. WT, wild type

\section{Author details}

${ }^{1}$ Advanced Center for Chronic Diseases (ACCDiS), Pontificia Universidad Católica de Chile, Santiago, Chile. ${ }^{2}$ Laboratory of Molecular Pathology, Department of Pathology, School of Medicine, BIOREN-CEGIN, and Graduate Program in Applied Cell and Molecular Biology, Universidad de La Frontera, Temuco, Chile. ${ }^{3}$ Center UC for Investigational in Oncology (CITO), Pontificia Universidad Católica de Chile, Santiago, Chile. ${ }^{4}$ Instituto de Química, Faculty of Science, Pontificia Universidad Católica de Valparaíso, Valparaiso, Chile. ${ }^{5}$ Advanced Center for Chronic Diseases (ACCDiS), Universidad de Chile, Santiago, Chile. ${ }^{6}$ Fundación Ciencia Y Vida, Parque Biotecnológico, Santiago, Chile. ${ }^{7}$ Department of Pathology, Faculty of Medicine, Hospital Clínico Universidad de Chile, Santiago, Chile. ${ }^{8}$ Department of Pathology, Faculty of Medicine, Pontificia Universidad Católica de Chile, Santiago, Chile. ${ }^{9}$ Esophagogastric Surgery Unit, Hospital Dr. Sótero del Río, Santiago, Chile. ${ }^{10}$ Digestive Surgery Department, Pontificia Universidad Católica de Chile, Santiago, Chile. ${ }^{11}$ Department of Physiology, Faculty of Biological Sciences, Pontificia Universidad Católica de Chile, Santiago, Chile. ${ }^{12}$ Department of Hematology-Oncology, Faculty of Medicine, Pontificia Universidad Católica de Chile, Santiago, Chile.
Published online: 08 March 2021

\section{Reference}

1. Sandoval-Bórquez A, Polakovicova I, Carrasco-Véliz N, Lobos-González L, Riquelme I, Carrasco-Avino G, Bizama C, Norero E, Owen GI, Roa JC, Corvalán AH. MicroRNA-335-5p is a potential suppressor of metastasis and invasion in gastric cancer. Clin Epigenet. 2017;9:114. https://doi. org/10.1186/s13148-017-0413-8.

\section{Publisher's Note}

Springer Nature remains neutral with regard to jurisdictional claims in published maps and institutional affiliations. 